\title{
Four and three-phonon scattering in isotropic superfluid helium
}

\author{
I.N. Adamenko ${ }^{1}$, Yu.A. Kitsenko ${ }^{2}$, K.E. Nemchenko ${ }^{1}$, and A.F.G. Wyatt ${ }^{3}$ \\ ${ }_{1}^{1}$ Karazin Kharkov National University, 4 Svobody Sq., Kharkov 61077, Ukraine \\ ${ }^{2}$ Akhiezer Institute for Theoretical Physics National Science Center "Kharkov Institute of Physics and Technology» \\ National Academy of Sciences of Ukraine, 1 Academicheskaya St., Kharkov 61108, Ukraine \\ ${ }^{3}$ School of Physics, University of Exeter, Exeter EX4 4QL, UK \\ E-mail: a.f.g.wyatt@exeter.ac.uk
}

Received October 28, 2008

\begin{abstract}
We analyse the important role of four-phonon processes ( $4 \mathrm{pp}$ ) in isotropic phonon systems of superfluid helium. The matrix elements and the rate of four-phonon processes are calculated. Special consideration is given to the $4 \mathrm{pp}$ in the momentum range where three-phonon processes are allowed. In this momentum range, we show that the $4 \mathrm{pp}$ scattering rate, at small angles, is equal to the scattering rate due to three-phonon processes. Then we show that the coefficient of first viscosity of superfluid helium is caused by two processes, the first is due to the transverse relaxation caused by many three-phonon processes and the second is due to four-phonon processes. The relaxation time that governs the viscosity is obtained from the sum of the rates from these two processes. The temperature dependence of the attenuation coefficient of a pulse of high-energy phonons in He II, due to scattering with thermal phonons, is also calculated. The theoretical results are compared with experimental data and found to be in good agreement.
\end{abstract}

PACS: 47.37.+q Hydrodynamic aspects of superfluidity; quantum fluids.

Keywords: superfluid helium, phonon scattering, dispersion curve, viscosity.

\section{Introduction}

Many of the properties of superfluid helium are described in terms of its excitations from the ground state, the phonons and rotons. There are interactions between these excitations which govern important characteristics such as the time to reach thermodynamic equilibrium, and the normal fluid viscosity. At low temperatures, $T<0.6 \mathrm{~K}$, there are practically no rotons so the phonons determine the behavior of superfluid ${ }^{4} \mathrm{He}$. Phonons mutually scatter by two main processes, three-phonon process ( $3 p p)$, in which one phonon decays into two phonons and vice versa, and four-phonon processes (4pp) in which two phonons scatter to two other phonons. Higher order processes are weak in comparision.

There is only one phonon branch in liquid helium, unlike solids which have transverse branches as well as a longitudinal branch. These branches in solids have normal dispersion, so $3 \mathrm{pp}$ in solids involve phonons from more than one branch. However 3 pp are allowed within the same branch in liquid helium, because the dispersion is anomalous over a large part of the phonon momentum range.

If we write the dispersion curve for phonons as

$$
\varepsilon=c p(1+\psi(p))
$$

where $c$ is the sound velocity, $\varepsilon$ and $p$ correspond to the energy and momentum of a phonon, then when $\psi(p)>0$, the dispersion is anomalous and when $\psi(p)<0$, the dispersion is normal and $3 \mathrm{pp}$ are not allowed. The function $|\psi(p)|<<1$ and it describes the deviation of the phonon spectrum from linearity. So we see that although the value of $\psi(p)$ is small, it completely determines the mechanisms of phonon interactions.

When $p<p_{c}$ (at the saturated vapour pressure $\left.\tilde{p}_{c}=c p_{c} / k_{B}=10 \mathrm{~K}\right) \psi(p)>0$ and the dispersion is anomalous. In this case, the conservation laws of energy and momentum allow processes which do not conserve phonon number. The fastest of these is the small-angle 
three-phonon process which has a typical rate $v_{3 p p}$ in which one phonon decays into two or two interacting phonons combine into one. When the angle between the momenta of the phonons is large, $>27^{\circ}$, three-phonon processes are forbidden by the conservation laws and the interaction between phonons is only by the slower four-phonon processes, with the typical rate $v_{4 p p}$. When $p>p_{c}$, function $\psi(p)<0$. In this case, the dispersion is normal and three-phonon processes are forbidden by the conservation laws of energy and momentum and the fastest scattering is by four-phonon processes.

There is a strong inequality between the typical values of the rates of three-phonon and four-phonon processes described above,

$$
v_{3 p p}>v_{4 p p}
$$

which causes dynamic systems of phonons, in superfluid helium, to separate into two subsystems: a subsystem of high-energy phonons (h-phonons) with $p>p_{c}$ in which equilibrium is attained relatively slowly and a subsystem of low-energy phonons (1-phonons) with $p<p_{c}$ in which equilibrium occurs relatively quickly (see, for example, Refs. 1-5).

The dissipative coefficients of superfluid helium are mainly governed by the interaction between 1-phonons. However $v_{3 p p}$ does not enter directly into the dissipative coefficients. Three-phonon processes cause thermal equilibrium to be established rapidly in a small angular range of momentum. Equilibrium over the whole angular range can be obtained in two ways. The first is by superdiffusion in space with the momentum vector having angular steps of a size which is typical of three-phonon processes. This has a typical rate $v_{\perp}$ that is three orders of magnitude lower than the three-phonon process rate (see, for example, Refs. 6-10). The second is by four-phonon processes (see, for example, Refs. 11 and 12), which can scatter through much larger angles than $3 \mathrm{pp}$. The rate from these important $4 \mathrm{pp}$ scatterings has not been obtained when there is anomalous dispersion which allows spontaneous decay.

Earlier rates were found indirectly from measurements of the dissipative coefficients of phonon systems. These relate to global equilibrium times. A direct measurement of the rate of four-phonon process scattering was made by measuring the attenuation of a pulse of h-phonon propagating through superfluid helium at a finite temperature [13]. Here the beam of $\mathrm{h}$-phonons is scattered by the isotropic distribution of thermal low-energy phonons. Recently, direct measurements of the phonon-phonon scattering rates interactions have been made by scattering two beams of phonons [14-16].

From the above we see that $4 p p$ scattering is fundamentally important to understanding some important properties of superfluid helium. In this paper we develop the detailed theory of $4 \mathrm{pp}$ scattering, including the momentum range where conservation laws of energy and momentum allow three-phonon processes. From the detailed theory we find numerical values for the scattering rates and the relaxation times in isotropic phonon systems. Hence we find the coefficient of first viscosity as a function of temperature. There is good agreement with the measured values.

In Sec. 2 we derive the matrix elements for $4 p p$, in Sec. 3 we find the relaxation in phonon system caused by four-phonon processes, in Sec. 4 we find the $4 p p$ rate when 3 pp are allowed, in Secs. 5 and 6 we calculate the relaxation time relevant to viscosity. In Sec. 7 we calculate the scattering of a beam of high-energy phonons by thermal phonons. Finally in Sec. 8 we draw conclusions.

\section{Matrix element for four-phonon processes with the account of possibility of three-phonon processes}

The interaction of phonons in superfluid helium can be described by the Landau Hamiltonian which can written as (see, for example, Ref. 17)

$$
\hat{H}_{\mathrm{ph}}=\hat{H}_{0}+\hat{V}_{3}+\hat{V}_{4},
$$

where $\hat{H}_{0}$ is the Hamiltonian of noninteracting phonons, terms $\hat{V}_{3}$ and $\hat{V}_{4}$ describe the interaction of phonons caused by small deviations of the system from equilibrium to third and fourth order, respectively.

The probability density of four-phonon process, following Refs. 13,17,18, can be written as

$$
W\left(\mathbf{p}_{1}, \mathbf{p}_{2} \mid \mathbf{p}_{3}, \mathbf{p}_{4}\right)=\frac{2 \pi}{\hbar} V^{2}\left|H_{f i}\right|^{2} \frac{1}{(2 \pi \hbar)^{6}},
$$

where $V$ is a volume of a system, $H_{f i}$ is the amplitude of four-phonon processes. This is obtained from $\hat{V}_{3}$ with second order perturbation theory, and from $\hat{V}_{4}$ with first order perturbation theory. Following standard procedures (see, for example, Refs. 13,17-20) we have

$$
\begin{aligned}
H_{f i}=\sum_{\mathbf{Q}} \frac{\left\langle\mathbf{p}_{3}, \mathbf{p}_{4}\left|\hat{V}_{3}\right| \mathbf{Q}\right\rangle\left\langle\mathbf{Q}\left|\hat{V}_{3}\right| \mathbf{p}_{1}, \mathbf{p}_{2}\right\rangle}{E_{i}-E_{\mathbf{Q}}}+ \\
\quad+\left\langle\mathbf{p}_{3}, \mathbf{p}_{4}\left|\hat{V}_{4}\right| \mathbf{p}_{1}, \mathbf{p}_{2}\right\rangle,
\end{aligned}
$$

where $E_{i}$ is the energy of initial state and $\mathbf{Q}$ is the intermediate state with energy $E_{\mathbf{Q}}$.

There are six possible intermediate states $|\mathbf{Q}\rangle$ :

$$
\begin{array}{ll}
\text { I. }\left|\mathbf{p}_{1}+\mathbf{p}_{2}\right\rangle ; & \text { II. }\left|\mathbf{p}_{2}, \mathbf{p}_{3}, \mathbf{p}_{1}-\mathbf{p}_{3}\right\rangle ; \\
\text { III. }\left|\mathbf{p}_{2}, \mathbf{p}_{4}, \mathbf{p}_{1}-\mathbf{p}_{4}\right\rangle ; & \text { IV. }\left|\mathbf{p}_{1}, \mathbf{p}_{3}, \mathbf{p}_{2}-\mathbf{p}_{3}\right\rangle ; \\
\text { V. }\left|\mathbf{p}_{1}, \mathbf{p}_{4}, \mathbf{p}_{2}-\mathbf{p}_{4}\right\rangle ; & \text { VI. }\left|\mathbf{p}_{1}, \mathbf{p}_{2}, \mathbf{p}_{3}, \mathbf{p}_{4},-\mathbf{p}_{1}-\mathbf{p}_{2}\right\rangle .
\end{array}
$$


There is an important effect when all four phonons participating in four-phonon process are 1-phonons. Then it is possible that the matrix elements diverge. This is because the denominator of the first term in Eq. (5) can be zero at some value of $\mathbf{Q}$. This happens when the transition from the initial state to the final state can be realized by two sequential three-phonon processes. This divergence can be eliminated if we take into account that the intermediate state has a finite lifetime. As pointed out in Ref. 21, the energy of a system which can decay into some quasi-stationary state, can be determined only to within an accuracy of $\Gamma=\hbar / \tau$, where $\tau$ is the lifetime of the system in this quasi-stationary state. In our case, the finiteness of the lifetime of the state $|\mathbf{Q}\rangle$ is due to the possibility that it can scatter by the three-phonon process. Thus $E_{\mathbf{Q}}$ in the denominator of the first term in Eq. (5), must be substituted by $E_{\mathbf{Q}}-i \Gamma^{(\mathbf{Q})}$, where $\Gamma^{(\mathbf{Q})}$ is defined by the lifetime of the corresponding intermediate state. The lifetimes of the corresponding intermediate states are

$$
\begin{array}{ll}
\text { I. } \tau_{(1)}=v_{d}^{-1}\left(\mathbf{p}_{1}+\mathbf{p}_{2}\right) ; & \text { II. } \tau_{(13)}=v_{c}^{-1}\left(\mathbf{p}_{1}-\mathbf{p}_{3}\right) ; \\
\text { III. } \tau_{(14)}=v_{c}^{-1}\left(\mathbf{p}_{1}-\mathbf{p}_{4}\right) ; & \text { IV. } \tau_{(23)}=v_{c}^{-1}\left(\mathbf{p}_{2}-\mathbf{p}_{3}\right) ; \\
\text { V. } \tau_{(24)}=v_{c}^{-1}\left(\mathbf{p}_{2}-\mathbf{p}_{4}\right) ; & \text { VI. } \tau_{(5)}=\infty .
\end{array}
$$

Here $v_{d}(\mathbf{p})$ is a rate of a decay of a phonon with momentum $\mathbf{p}$ into two, and $v_{c}(\mathbf{p})$ is the rate of the three-phonon processes in which a phonon with momentum $\mathbf{p}$ combines with the other phonon. These $3 p p$ rates were calculated by us in Ref. 4 for isotropic and anisotropic phonon systems. The intermediate state VI cannot be realized by three-phonon processes and therefore the denominator cannot be zero at any value of momentum. We therefore consider that the lifetime of such a state is infinite and the value of $\Gamma$ is $\Gamma=\hbar / \tau=0$. If one of the four interacting phonons is an h-phonon, then there are no divergences in the first term of Eq. (5). In this case all lifetimes in Eq. (7) should be considered infinite, and all $\Gamma$ 's equal to 0 .

From relations (5) and (6) it follows that

$$
H_{f i}=\frac{\sqrt{p_{1} p_{2} p_{3} p_{4}}}{8 \rho V} M_{\Sigma} .
$$

Here $\rho=145 \mathrm{~kg} / \mathrm{m}^{3}$ is a density of He II, and

$$
M_{\Sigma}=M^{(1)}+M_{13}^{(3)}+M_{14}^{(3)}+M_{23}^{(3)}+M_{24}^{(3)}+M^{(5)}+M_{4}
$$

is a matrix element which consists of seven terms, six of which correspond to the six intermediate states (6), and the seventh is determined by the contribution of $\hat{V}_{4}$ to first order. We can write these terms as

$$
\begin{aligned}
& M^{(1)}=\frac{\varepsilon_{1+2}}{\varepsilon_{1}+\varepsilon_{2}-\varepsilon_{1+2}+i \Gamma^{(1)}} \times \\
& \times\left(2 u-1+\mathbf{n}_{1} \mathbf{n}_{2}+\mathbf{n}_{1} \mathbf{n}_{1+2}+\mathbf{n}_{2} \mathbf{n}_{1+2}\right) \times \\
& \times\left(2 u-1+\mathbf{n}_{3} \mathbf{n}_{4}+\mathbf{n}_{3} \mathbf{n}_{3+4}+\mathbf{n}_{4} \mathbf{n}_{3+4}\right), \\
& M^{(5)}=-\frac{\varepsilon_{1+2}}{\varepsilon_{1}+\varepsilon_{2}+\varepsilon_{1+2}}\left(2 u-1+\mathbf{n}_{1} \mathbf{n}_{2}-\mathbf{n}_{1} \mathbf{n}_{1+2}-\mathbf{n}_{2} \mathbf{n}_{1+2}\right) \times \\
& \times\left(2 u-1+\mathbf{n}_{3} \mathbf{n}_{4}-\mathbf{n}_{3} \mathbf{n}_{3+4}-\mathbf{n}_{4} \mathbf{n}_{3+4}\right), \\
& M_{4}=4\left\{(u-1)^{2}+w\right\}, \\
& M_{13}^{(3)}=\frac{\varepsilon_{1-3}}{\varepsilon_{1}-\varepsilon_{3}-\varepsilon_{1-3}+i \Gamma^{(13)}} \times \\
& \times\left(2 u-1+\mathbf{n}_{1} \mathbf{n}_{3}+\mathbf{n}_{1} \mathbf{n}_{1-3}+\mathbf{n}_{3} \mathbf{n}_{1-3}\right) \times \\
& \times\left(2 u-1+\mathbf{n}_{2} \mathbf{n}_{4}+\mathbf{n}_{2} \mathbf{n}_{1-3}+\mathbf{n}_{4} \mathbf{n}_{1-3}\right) .
\end{aligned}
$$

Here $\mathbf{n}_{i}=\mathbf{p}_{i} / p_{i}, \Gamma^{(q)}=\hbar \tau_{(q)}^{-1}, u=(\rho / c) \partial c / \partial \rho=2.84$ is a Grüneisen constant, $w=\left(\rho^{2} / c\right) \partial^{2} c / \partial \rho^{2}=0.188, \varepsilon_{i}=\varepsilon\left(\mathbf{p}_{i}\right)$. The rest of the terms in Eq. (9), i.e., $M_{14}^{(3)}, M_{23}^{(3)}$ and $M_{24}^{(3)}$ can be obtained from $M_{13}^{(3)}$ by the replacement of the corresponding subscripts. The matrix element is given in detail in Ref. 22, to which the corresponding $\Gamma$ 's should be added (see Eqs. (10) and (13)).

\section{Relaxation in phonon system caused by four-phonon processes}

We consider four-phonon process which have two phonons in the initial state and two phonons in the final state. The conservation of energy and momentum is expressed as

$$
\varepsilon_{1}+\varepsilon_{2}=\varepsilon_{3}+\varepsilon_{4}, \quad \mathbf{p}_{1}+\mathbf{p}_{2}=\mathbf{p}_{3}+\mathbf{p}_{4} .
$$

The kinetic equation describing the change of the distribution function $n\left(\mathbf{p}_{1}\right)=n_{1}$ due to $4 \mathrm{pp}$ scattering is

$$
\frac{d n_{1}}{d t}=N_{b}\left(\mathbf{p}_{1}\right)-N_{d}\left(\mathbf{p}_{1}\right)
$$

where $N_{b}\left(\mathbf{p}_{1}\right)$ and $N_{d}\left(\mathbf{p}_{1}\right)$ are the respective rates of increasing and decreasing number of phonons with momentum $\mathbf{p}_{1}$ in unit time due to collisions. They can be written as

$$
\begin{gathered}
N_{b, d}\left(\mathbf{p}_{1}\right)= \\
=\frac{1}{2} \int W\left(\mathbf{p}_{1}, \mathbf{p}_{2} \mid \mathbf{p}_{3}, \mathbf{p}_{4}\right) \delta\left(\mathbf{p}_{\Sigma}\right) \delta\left(\varepsilon_{\Sigma}\right) n_{b, d} d^{3} p_{2} d^{3} p_{3} d^{3} p_{4} .
\end{gathered}
$$

The probability density $W\left(\mathbf{p}_{1}, \mathbf{p}_{2} \mid \mathbf{p}_{3}, \mathbf{p}_{4}\right)$ is defined by Eq. (4) and determines the probability of the process. The factor $1 / 2$ is due to the identity of processes 
$\mathbf{p}_{1}+\mathbf{p}_{2} \leftrightarrow \mathbf{p}_{3}+\mathbf{p}_{4}$ and $\mathbf{p}_{1}+\mathbf{p}_{2} \leftrightarrow \mathbf{p}_{4}+\mathbf{p}_{3}$, the $\delta$-functions correspond to the conservation laws of energy $\varepsilon_{\Sigma}=$ $=\varepsilon_{1}+\varepsilon_{2}-\varepsilon_{3}-\varepsilon_{4}$ and momentum $\mathbf{p}_{\Sigma}=\mathbf{p}_{1}+\mathbf{p}_{2}-\mathbf{p}_{3}-\mathbf{p}_{4}$, and

$$
n_{b}=n_{3} n_{4}\left(1+n_{2}\right)\left(1+n_{1}\right), \quad n_{d}=n_{1} n_{2}\left(1+n_{3}\right)\left(1+n_{4}\right) .
$$

To determine the typical rate of four-phonon processes in phonon systems in superfluid helium, we take the distribution functions in Eq. (15) as

$$
n_{1}=n_{1}^{(0)}+\delta n_{1}, n_{2}=n_{2}^{(0)}, n_{3}=n_{3}^{(0)}, n_{4}=n_{4}^{(0)} .
$$

In Eq. (18) superscript «0» corresponds to the equilibrium distribution function, and $\delta n$ is the deviation of the distribution function from equilibrium.

The equilibrium distribution function of phonons, according to Refs. 4, 23, can be written as

$$
n^{(0)}(\mathbf{p})=\left\{\exp \left(\frac{\varepsilon-\mathbf{p u}}{k_{B} T}\right)-1\right\}^{-1},
$$

where

$$
\mathbf{u}=\mathbf{N} c(1-\chi)
$$

is a drift velocity, $\mathbf{N}$ is a unit vector directed along the total momentum of phonon system. This defines an anisotropy axis of phonon system and $\chi$ is the anisotropy parameter.

For isotropic systems the parameter $\chi=1$ and $u=0$ in Eq. (19). For weakly anisotropic systems $\chi$ is close to 1 . However for strongly anisotropic phonon systems $\chi<<1$. Such systems can be created experimentally [14-16].

The typical rate of relaxation caused by four-phonon processes $v_{4 p p}$, according to kinetic theory in the relaxation time approximation, can be defined as

$$
v_{4 p p}\left(\mathbf{p}_{1}\right)=-\frac{1}{\delta n_{1}} \frac{d \delta n_{1}}{d t} .
$$

From Eqs. (15)-( 21) we have

$$
v_{4 p p}\left(\mathbf{p}_{1}\right)=\frac{1}{2} \frac{1}{1+n_{1}^{(0)}} \int d^{3} p_{2} d^{3} p_{3} d^{3} p_{4} \times
$$

$$
\times W\left(\mathbf{p}_{1}, \mathbf{p}_{2} \mid \mathbf{p}_{3}, \mathbf{p}_{4}\right) \delta\left(\mathbf{p}_{\Sigma}\right) \delta\left(\varepsilon_{\Sigma}\right) n_{2}^{(0)}\left(1+n_{3}^{(0)}\right)\left(1+n_{4}^{(0)}\right) .
$$

Equation (22) can be integrated with a help of the $\delta$-functions. For this we rewrite Eq. (22) taking into account (4), (8)-(13) in a spherical coordinate system

$$
\begin{gathered}
v_{4 p p}=\frac{p_{1}}{2^{11} \pi^{5} \hbar^{7} \rho^{2}} \frac{1}{1+n_{1}^{(0)}} \times \\
\times \int d p_{2} d \varphi_{2} d \zeta_{2} d p_{3} d \varphi_{3} d \zeta_{3} d p_{4} d \varphi_{4} d \zeta_{4} p_{2}^{3} p_{3}^{3} p_{4}^{3} \times \\
\times M_{\Sigma}^{2} \delta\left(\mathbf{p}_{\Sigma}\right) \delta\left(\varepsilon_{\Sigma}\right) n_{2}^{(0)}\left(1+n_{3}^{(0)}\right)\left(1+n_{4}^{(0)}\right)
\end{gathered}
$$

where $\zeta_{i}=1-\left(\mathbf{p}_{i} \mathbf{N} / p_{i}\right)$ and $\varphi_{i}$ is the azimuth angle of the phonon with momentum $\mathbf{p}_{i}$. Without loss of generality, we assume that $p_{3}>p_{4}$ for the integration.

Making the integration in Eq. (23), with a help of the $\delta$-functions (see Appendix A) we have

$$
\begin{gathered}
v_{4 p p}=\frac{1}{2^{10} \pi^{5} \hbar^{7} \rho^{2} c} \frac{p_{1}}{1+n_{1}^{(0)}} \times \\
\times \int d p_{2} d p_{3} d \zeta_{2} d \zeta_{3} d \varphi_{2} \frac{p_{2}^{3} p_{3}^{3} p_{4}^{2}}{\sqrt{R}} \times \\
\times\left\{M_{(+)}^{2}+M_{(-)}^{2}\right\} n_{2}^{(0)}\left(1+n_{3}^{(0)}\right)\left(1+n_{4}^{(0)}\right),
\end{gathered}
$$

where

$$
\begin{gathered}
p_{4}=p_{1}+p_{2}-p_{3}-\phi, \quad \zeta_{4}=\frac{p_{1} \zeta_{1}+p_{2} \zeta_{2}-p_{3} \zeta_{3}-\phi}{p_{4}} \\
\phi=p_{3} \psi_{3}+p_{4} \psi_{4}-p_{1} \psi_{1}-p_{2} \psi_{2}, \quad \psi_{i}=\psi\left(p_{i}\right), \\
M_{( \pm)}=M_{\Sigma}\left(\cos \varphi_{3}=\cos \varphi_{3}^{( \pm)}, \cos \varphi_{4}=\cos \varphi_{4}^{( \pm)}\right)
\end{gathered}
$$

and $\cos \varphi_{3,4}^{( \pm)}$are determined by Eqs. (88), (89) of Appen$\operatorname{dix}$ A.

Further integration of Eq. (24) cannot be precisely made analytically, because of the complexity of the integrand. The results of a numerical calculation of the 1-phonon relaxation rate from Eq. (24), for the isotropic case at temperature $T=0.6 \mathrm{~K}$, are shown in Fig. 1, curve 1 .

The limits of integration in Eq. (24), with the condition $p_{3}>p_{4}$, are the momenta given by

$$
\begin{gathered}
p_{3 \text { low }}=\frac{p_{1}+p_{2}}{2}, \quad p_{3 \text { up }}=\min \left(p_{1}+p_{2}, p_{c}\right), \\
p_{\text {2low }}=0, \quad p_{2 \text { up }}=p_{c} .
\end{gathered}
$$

For comparison, the rate of three-phonon processes in the isotropic case with $T=0.6 \mathrm{~K}$ calculated by us in Ref. 4 is shown by curve 5 .

From Fig. 1, it can be seen that in practically all the momentum range, where three-phonon processes are allowed, the rate of four-phonon processes is almost the same as the rate of three-phonon processes. To further understand this result, the contribution of the different angular groups of phonons with momentum $\mathbf{p}_{2}$ is calculated. 


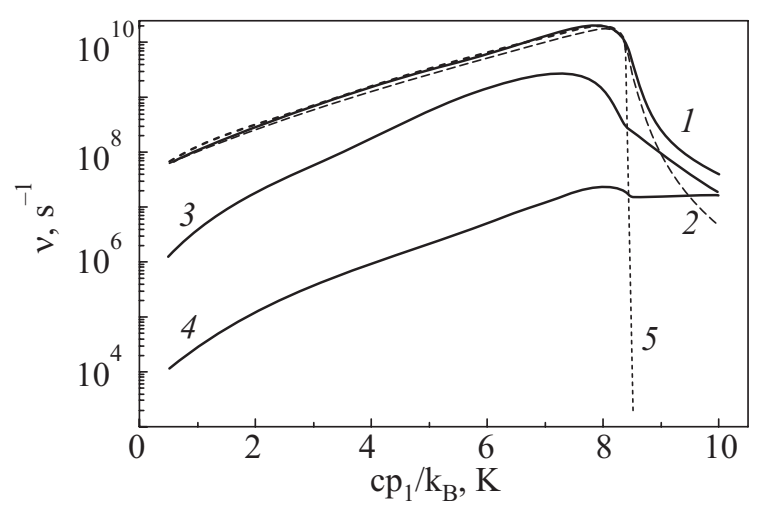

Fig. 1. The rate of four-phonon processes in the isotropic 1-phonon system with $T=0.6 \mathrm{~K}$ (curve 1 ) as a function of momentum $\mathbf{p}_{1}$; curves $2-4$ show contributions to the relaxation rate by phonons $\mathbf{p}_{2}$ having angles with phonon $\mathbf{p}_{1}: 0-30^{\circ}$ (curve 2 ), $30-60^{\circ}$ (curve 3 ) and $60-180^{\circ}$ (curve 4 ). Curve 5 shows the rate of three-phonon processes in the isotropic case for $T=0.6 \mathrm{~K}$. It can be seen that curves 1 and 5 are almost coincident for $p_{1}<p_{c}$.

Curves 2-4 show the contributions to the rate of phonons with momentum $\mathbf{p}_{2}$ having angles with a phonon $\mathbf{p}_{1}$ : $0-30^{\circ}$ (curve 2), $30-60^{\circ}$ (curve 3 ) and 60-180 (curve 4). From Fig. 1 it can be seen, that the main contribution to the rate of four-phonon processes involves phonons with momentum $\mathbf{p}_{2}$, at an angle up to $30^{\circ}$ to phonon $\mathbf{p}_{1}$. We conclude that the rate of these four-phonon processes is practically equal to the rate of three-phonon processes. From this analysis, it follows that the main contribution to $v_{4 p p}$, where three-phonon processes are allowed, is caused by small angle scattering, and then $v_{4 p p}$ appears practically equal to $v_{3 p p}$.

In order to understand why there is a coincidence between $v_{3 p p}$ and $v_{4 p p}$ for angles where three-phonon processes are permitted, it is necessary to make analytical calculations which we do in the next section.

\section{The rate of four-phonon processes in the range of angles where three-phonon processes are permitted}

To calculate the rate of four-phonon processes, at small angles where three-phonon processes are allowed, we start from Eq. (22) for the rate and Eq. (4) for the probability density of the four-phonon processes. At small angles, the main contribution to the matrix element (9), is given by the five resonant terms corresponding to the intermediate states I-V (see (6)). Our calculations show that after squaring the absolute value of the matrix element, cross-terms give a small contribution to the integral. So the main contribution is caused by the squares of the five resonant terms.

We now calculate the contribution to the rate of each of the five terms mentioned above. The probability density
$W_{I}$ of the four-phonon process which is caused by the intermediate state $I$, is conveniently written in the form

$$
\begin{gathered}
W_{\mathrm{I}}\left(\mathbf{p}_{1}, \mathbf{p}_{2} \mid \mathbf{p}_{3}, \mathbf{p}_{4}\right)=\frac{2 \pi}{\hbar} V^{2} \frac{1}{(2 \pi \hbar)^{6}} \times \\
\times\left.\int \frac{\left\langle\mathbf{p}_{3}, \mathbf{p}_{4}\left|\hat{V}_{3}\right| \mathbf{q}\right\rangle\left\langle\mathbf{q}\left|\hat{V}_{3}\right| \mathbf{p}_{1}, \mathbf{p}_{2}\right\rangle}{\varepsilon_{1}+\varepsilon_{2}-\varepsilon_{\mathbf{q}}+i \Gamma_{d}(\mathbf{q})}\right|^{2} \delta\left(\mathbf{p}_{3}+\mathbf{p}_{4}-\mathbf{q}\right) d^{3} q,
\end{gathered}
$$

where

$$
\Gamma_{d}(\mathbf{q})=\hbar v_{d}(\mathbf{q})
$$

The probability density of three-phonon processes is given by (see, for example, Ref. 4)

$$
W\left(\mathbf{p}_{i}, \mathbf{p}_{j} \mid \mathbf{q}\right)=\frac{2 \pi}{\hbar} V\left|\left\langle\mathbf{p}_{i}, \mathbf{p}_{j}\left|\hat{V}_{3}\right| \mathbf{q}\right\rangle\right|^{2} .
$$

Taking (32) into account, we can rewrite Eq. (30) as

$$
\begin{gathered}
W_{\mathrm{I}}\left(\mathbf{p}_{1}, \mathbf{p}_{2} \mid \mathbf{p}_{3}, \mathbf{p}_{4}\right)=\frac{\hbar}{2 \pi} \frac{1}{(2 \pi \hbar)^{6}} \times \\
\times \int \frac{W\left(\mathbf{p}_{3}, \mathbf{p}_{4} \mid \mathbf{q}\right) W\left(\mathbf{q} \mid \mathbf{p}_{1}, \mathbf{p}_{2}\right)}{\left(\varepsilon_{1}+\varepsilon_{2}-\varepsilon_{\mathbf{q}}\right)^{2}+\Gamma_{d}^{2}(\mathbf{q})} \delta\left(\mathbf{p}_{3}+\mathbf{p}_{4}-\mathbf{q}\right) d^{3} q .
\end{gathered}
$$

As

$$
\begin{gathered}
\frac{1}{\left(\varepsilon_{1}+\varepsilon_{2}-\varepsilon_{\mathbf{q}}\right)^{2}+\Gamma_{d}^{2}(\mathbf{q})}= \\
=\frac{\pi}{\Gamma_{d}(\mathbf{q})} \frac{\Gamma_{d}(\mathbf{q})}{\pi\left\{\left(\varepsilon_{1}+\varepsilon_{2}-\varepsilon_{\mathbf{q}}\right)^{2}+\Gamma_{d}^{2}(\mathbf{q})\right\}},
\end{gathered}
$$

then for small values of $\Gamma$, due to the equality

$$
\lim _{\gamma \rightarrow 0} \frac{\gamma}{\pi\left(\alpha^{2}+\gamma^{2}\right)}=\delta(\alpha)
$$

the second factor in the right-hand side of Eq. (34) can be approximately replaced by a $\delta$-function.

Substituting expression (34) into (33), taking into account (35), we obtain

$$
\begin{gathered}
W_{\mathrm{I}}\left(\mathbf{p}_{1}, \mathbf{p}_{2} \mid \mathbf{p}_{3}, \mathbf{p}_{4}\right)=\frac{\hbar}{2} \frac{1}{(2 \pi \hbar)^{6}} \times \\
\times \int \frac{W\left(\mathbf{p}_{3}, \mathbf{p}_{4} \mid \mathbf{q}\right) W\left(\mathbf{q} \mid \mathbf{p}_{1}, \mathbf{p}_{2}\right)}{\Gamma_{d}(\mathbf{q})} \delta\left(\varepsilon_{1}+\varepsilon_{2}-\varepsilon_{\mathbf{q}}\right) \delta\left(\mathbf{p}_{3}+\mathbf{p}_{4}-\mathbf{q}\right) d^{3} q
\end{gathered}
$$

Then substituting (36) into (22) and assuming the angles are small, we have 


$$
\begin{gathered}
v_{4 p p}^{\mathrm{I}}\left(\mathbf{p}_{1}\right)=\frac{\hbar}{4} \frac{1}{(2 \pi \hbar)^{6}} \frac{1}{1+n_{1}^{(0)}} \int d^{3} q d^{3} p_{2} d^{3} p_{3} d^{3} p_{4} \times \\
\times \frac{W\left(\mathbf{p}_{3}, \mathbf{p}_{4} \mid \mathbf{q}\right) W\left(\mathbf{q} \mid \mathbf{p}_{1}, \mathbf{p}_{2}\right)}{\Gamma_{d}(\mathbf{q})} n_{2}^{(0)}\left(1+n_{3}^{(0)}\right)\left(1+n_{4}^{(0)}\right) \times
\end{gathered}
$$$$
\times \delta\left(\mathbf{p}_{1}+\mathbf{p}_{2}-\mathbf{q}\right) \delta\left(\mathbf{p}_{3}+\mathbf{p}_{4}-\mathbf{q}\right) \delta\left(\varepsilon_{1}+\varepsilon_{2}-\varepsilon_{\mathbf{q}}\right) \delta\left(\varepsilon_{3}+\varepsilon_{4}-\varepsilon_{\mathbf{q}}\right) .
$$

Taking the $\delta$-functions into account, together with the properties of the equilibrium distribution functions, the combination of distribution functions in Eq. (37) can be rewritten in the form

$$
\begin{aligned}
& \frac{1}{1+n_{1}^{(0)}} n_{2}^{(0)}\left(1+n_{3}^{(0)}\right)\left(1+n_{4}^{(0)}\right)= \\
& =\left(n_{2}^{(0)}-n_{\mathbf{q}}^{(0)}\right)\left(1+n_{3}^{(0)}+n_{4}^{(0)}\right) .
\end{aligned}
$$

The rate of decay of a phonon with momentum $\mathbf{p}_{i}$, due to the three-phonon process $\mathbf{p}_{i}=\mathbf{p}_{j}+\mathbf{p}_{k}$ is, according to Ref. 4, equal to

$$
\begin{aligned}
v_{d}\left(\mathbf{p}_{i}\right)=\frac{1}{2} \int & d^{3} p_{j} d^{3} p_{k} \frac{W\left(\mathbf{p}_{i} \mid \mathbf{p}_{j}, \mathbf{p}_{k}\right)}{(2 \pi \hbar)^{3}}\left(1+n_{j}^{(0)}+n_{k}^{(0)}\right) \times \\
& \times \delta\left(\mathbf{p}_{i}-\mathbf{p}_{j}-\mathbf{p}_{k}\right) \delta\left(\varepsilon_{i}-\varepsilon_{j}-\varepsilon_{k}\right)
\end{aligned}
$$

and the rate of combining with the phonon with momen$\operatorname{tum} \mathbf{p}_{j}$ is

$$
\begin{aligned}
v_{c}\left(\mathbf{p}_{j}\right)= & \int d^{3} p_{k} d^{3} p_{i} \frac{W\left(\mathbf{p}_{j}, \mathbf{p}_{k} \mid \mathbf{p}_{i}\right)}{(2 \pi \hbar)^{3}}\left(n_{k}^{(0)}-n_{i}^{(0)}\right) \times \\
& \times \delta\left(\mathbf{p}_{j}+\mathbf{p}_{k}-\mathbf{p}_{i}\right) \delta\left(\varepsilon_{j}+\varepsilon_{k}-\varepsilon_{i}\right) .
\end{aligned}
$$

Taking into account the equality $W\left(\mathbf{p}_{i} \mid \mathbf{p}_{j}, \mathbf{p}_{k}\right)=$ $=W\left(\mathbf{p}_{j}, \mathbf{p}_{k} \mid \mathbf{p}_{i}\right)$ we have

$$
\begin{aligned}
v_{4 p p}^{\mathrm{I}}\left(\mathbf{p}_{1}\right)= & \frac{\hbar}{2(2 \pi \hbar)^{3}} \int d^{3} q d^{3} p_{2}\left(n_{2}^{(0)}-n_{\mathbf{q}}^{(0)}\right) \frac{W\left(\mathbf{q} \mid \mathbf{p}_{1}, \mathbf{p}_{2}\right)}{\Gamma_{d}(\mathbf{q})} \times \\
& \times v_{d}(\mathbf{q}) \delta\left(\mathbf{p}_{1}+\mathbf{p}_{2}-\mathbf{q}\right) \delta\left(\varepsilon_{1}+\varepsilon_{2}-\varepsilon_{\mathbf{q}}\right) .
\end{aligned}
$$

On substituting (31) into (41) and taking (40) into account, we finally have

$$
v_{4 p p}^{\mathrm{I}}\left(\mathbf{p}_{1}\right)=\frac{1}{2} v_{c}\left(\mathbf{p}_{1}\right)
$$

Now we calculate the contribution to rate due to the intermediate state II. The probability density of a four-phonon process caused by the intermediate state II is conveniently written in the form

$$
\begin{gathered}
W_{\mathrm{II}}\left(\mathbf{p}_{1}, \mathbf{p}_{2} \mid \mathbf{p}_{3}, \mathbf{p}_{4}\right)=\frac{2 \pi}{\hbar} V^{2} \frac{1}{(2 \pi \hbar)^{6}} \times \\
\times \int\left|\frac{\left\langle\mathbf{p}_{3}, \mathbf{p}_{4}\left|\hat{V}_{3}\right| \mathbf{q}, \mathbf{p}_{2}, \mathbf{p}_{3}\right\rangle\left\langle\mathbf{q}, \mathbf{p}_{2}, \mathbf{p}_{3}\left|\hat{V}_{3}\right| \mathbf{p}_{1}, \mathbf{p}_{2}\right\rangle}{\varepsilon_{1}-\varepsilon_{\mathbf{q}}-\varepsilon_{3}+i \Gamma_{c}(\mathbf{q})}\right|^{2} \times \\
\times \delta\left(\mathbf{p}_{1}-\mathbf{q}-\mathbf{p}_{3}\right) d^{3} q
\end{gathered}
$$

where

$$
\Gamma_{c}(\mathbf{q})=\hbar v_{c}(\mathbf{q})
$$

Taking (32) into account we can rewrite (43) as

$$
\begin{gathered}
W_{\mathrm{II}}\left(\mathbf{p}_{1}, \mathbf{p}_{2} \mid \mathbf{p}_{3}, \mathbf{p}_{4}\right)=\frac{\hbar}{2 \pi} \frac{1}{(2 \pi \hbar)^{6}} \times \\
\times \int \frac{W\left(\mathbf{p}_{4} \mid \mathbf{q}, \mathbf{p}_{2}\right) W\left(\mathbf{q}, \mathbf{p}_{3} \mid \mathbf{p}_{1}\right)}{\left(\varepsilon_{1}-\varepsilon_{\mathbf{q}}-\varepsilon_{3}\right)^{2}+\Gamma_{c}^{2}(\mathbf{q})} \delta\left(\mathbf{p}_{1}-\mathbf{q}-\mathbf{p}_{3}\right) d^{3} q .
\end{gathered}
$$

Replacing the resonant factor in Eq. (45) by a $\delta$-function, in a similar way to the previous case, we obtain

$$
\begin{gathered}
W_{\mathrm{II}}\left(\mathbf{p}_{1}, \mathbf{p}_{2} \mid \mathbf{p}_{3}, \mathbf{p}_{4}\right)=\frac{\hbar}{2} \frac{1}{(2 \pi \hbar)^{6}} \times \\
\times \int \frac{W\left(\mathbf{p}_{4} \mid \mathbf{q}, \mathbf{p}_{2}\right) W\left(\mathbf{q}, \mathbf{p}_{3} \mid \mathbf{p}_{1}\right)}{\Gamma_{c}(\mathbf{q})} \delta\left(\varepsilon_{1}-\varepsilon_{\mathbf{q}}-\varepsilon_{3}\right) \delta\left(\mathbf{p}_{1}-\mathbf{q}-\mathbf{p}_{3}\right) d^{3} q .
\end{gathered}
$$

Substituting (46) into (22), and assuming the angles are small, we have

$$
\begin{gathered}
v_{4 p p}^{\mathrm{II}}\left(\mathbf{p}_{1}\right)=\frac{\hbar}{4} \frac{1}{(2 \pi \hbar)^{6}} \frac{1}{1+n_{1}^{(0)}} \int d^{3} q d^{3} p_{2} d^{3} p_{3} d^{3} p_{4} n_{2}^{(0)} \times \\
\times\left(1+n_{3}^{(0)}\right)\left(1+n_{4}^{(0)}\right) \frac{W\left(\mathbf{p}_{4} \mid \mathbf{q}, \mathbf{p}_{2}\right) W\left(\mathbf{q}, \mathbf{p}_{3} \mid \mathbf{p}_{1}\right)}{\Gamma_{c}(\mathbf{q})} \delta\left(\mathbf{p}_{1}-\mathbf{q}-\mathbf{p}_{3}\right) \times \\
\times \delta\left(\mathbf{p}_{4}-\mathbf{q}-\mathbf{p}_{2}\right) \delta\left(\varepsilon_{1}-\varepsilon_{\mathbf{q}}-\varepsilon_{3}\right) \delta\left(\varepsilon_{4}-\varepsilon_{\mathbf{q}}-\varepsilon_{2}\right)
\end{gathered}
$$

Taking the $\delta$-functions and the properties of the equilibrium distribution functions into account, the combination of distribution functions in the integral can be rewritten in the form

$$
\begin{aligned}
& \frac{1}{1+n_{1}^{(0)}} n_{2}^{(0)}\left(1+n_{3}^{(0)}\right)\left(1+n_{4}^{(0)}\right)= \\
& =\left(n_{2}^{(0)}-n_{4}^{(0)}\right)\left(1+n_{3}^{(0)}+n_{\mathbf{q}}^{(0)}\right)
\end{aligned}
$$

Taking (48) into account we can rewrite (47) as

$$
\begin{aligned}
& v_{4 p p}^{\mathrm{II}}\left(\mathbf{p}_{1}\right)=\frac{\hbar}{4(2 \pi \hbar)^{3}} \int d^{3} q d^{3} p_{3}\left(1+n_{3}^{(0)}+n_{\mathbf{q}}^{(0)}\right) \times \\
& \times \frac{W\left(\mathbf{q}, \mathbf{p}_{3} \mid \mathbf{p}_{1}\right)}{\Gamma_{c}(\mathbf{q})} v_{c}(\mathbf{q}) \delta\left(\mathbf{p}_{1}-\mathbf{q}-\mathbf{p}_{3}\right) \delta\left(\varepsilon_{1}-\varepsilon_{\mathbf{q}}-\varepsilon_{3}\right) .
\end{aligned}
$$


Having substituted (44) into (49) we finally have

$$
v_{4 p p}^{\mathrm{II}}\left(\mathbf{p}_{1}\right)=\frac{1}{2} v_{d}\left(\mathbf{p}_{1}\right)
$$

We draw the reader's attention to an apparent contradiction. Firstly when $n_{2}^{(0)}$ tends to zero in Eq. (47), then it seems that the rate $v_{4 p p}^{\text {II }}\left(\mathbf{p}_{1}\right)$, also tends to zero too. Secondly, the rate of decay $v_{d}\left(\mathbf{p}_{1}\right)$ does not tend to zero when $n_{2}^{(0)}$ goes to zero. So we arrive at the contradiction that the left-hand side of Eq. (50) is equal to zero while the right-hand side is nonzero. However, if $n_{2}^{(0)}$ goes to zero, then $\Gamma_{c}(\mathbf{q})$ goes to zero too. So after cancelling $n_{2}^{(0)}$ with the same term in the expression for $\Gamma_{c}$ (see Eqs. (44) and (40)) we find that the rate $v_{4 p p}^{\text {II }}\left(\mathbf{p}_{1}\right)$ is nonzero. Consequently we are convinced in correctness of Eq. (50), which is valid at all values of $n_{2}^{(0)}$ which are not exactly zero.

The calculation of $v_{4} \mathrm{II}_{\mathrm{II}}\left(\mathbf{p}_{1}\right)$ can be made in a way similar to the calculation of $v_{4 p p}$ II $\left(\mathbf{p}_{1}\right)$, if we replace $\mathbf{p}_{3} \leftrightarrow \mathbf{p}_{4}$ in the integrand. Thus we obtain

$$
v_{4 p p}^{\text {III }}\left(\mathbf{p}_{1}\right)=\frac{1}{2} v_{d}\left(\mathbf{p}_{1}\right) .
$$

Analogous calculations for the fourth and fifth intermediate states give

$$
v_{4 p p}^{\mathrm{IV}}\left(\mathbf{p}_{1}\right)=v_{4 p p}^{\mathrm{V}}\left(\mathbf{p}_{1}\right)=\frac{1}{4} v_{c}\left(\mathbf{p}_{1}\right)
$$

Finally we have

$$
v_{4 p p}\left(\mathbf{p}_{1}\right)=v_{c}\left(\mathbf{p}_{1}\right)+v_{d}\left(\mathbf{p}_{1}\right)=v_{3 p p}\left(\mathbf{p}_{1}\right) .
$$

Thus, the rate of four-phonon processes is equal to the rate of three-phonon processes, in the momentum range where three-phonon processes are allowed.

Thus, for small angle scattering, the main contribution to the $4 \mathrm{pp}$ rate is caused by processes which can be represented as two consecutive three-phonon processes. At larger angles between the momenta of phonons $\mathbf{p}_{1}$ and $\mathbf{p}_{2}$, this mechanism becomes forbidden by the conservation laws of energy and momentum and the scattering is caused by «exclusive» four-phonon processes. These processes exclude 4pp which can be represented by two consequative $3 \mathrm{pp}$. As a result, the scattering rate obtained by us is the sum of the three-phonon process rate at small angles $\left(\lesssim 30^{\circ}\right)$, and the rate of «exclusive» four-phonon processes at larger angles.

\section{The calculation of the viscous relaxation time}

In this section we calculate the relaxation time that determines the coefficient of first viscosity of superfluid helium. We start from the stationary kinetic equation for phonons. Following Ref. 12, we consider a macroscopic nonuniform flow of fluid with velocity $\mathbf{u}(\mathbf{r})$ directed along $z$ axis. The velocity gradient is taken to be sufficiently small so that in every moving volume element, there is a local equilibrium. We consider that the velocity gradient is directed along the $x$ axis which is perpendicular to the $z$ axis. The distribution function $n_{i}$ of phonons with momentum $\mathbf{p}_{i}$ can be represented as a sum of a local-equilibrium distribution function $n_{i}^{(0)}$, and a small deviation $\delta n_{i}$ :

$$
n_{i}=n_{i}^{(0)}+\delta n_{i}
$$

Taking all the above into account, the kinetic equation can be written as

$$
n_{1}^{(0)}\left(n_{1}^{(0)}+1\right) \frac{c p}{k_{B} T} \frac{\partial u}{\partial x} \cos \theta \sin \theta \cos \varphi=I\left(n_{1}\right)
$$

where $\theta$ is the polar angle between the phonon momentum and the $z$ axis, and $\varphi$ is the azimuth angle of the phonon momentum relative to the $x$ axis.

The collision integral, in the relaxation time $\tau$ approximation, can be written as

$$
I\left(n_{1}\right)=-\frac{\delta n_{1}}{\tau}
$$

From the kinetic equation (55), the deviation of the distribution function from its equilibrium value, which is caused by the macroscopic velocity gradient, can be written as

$$
\delta n_{i}=n_{i}^{(0)}\left(n_{i}^{(0)}+1\right) \frac{P_{2}\left(\cos \theta_{i}\right)}{k_{B} T}\left(a \varepsilon_{i}+b c p_{i}\right),
$$

where $P_{2}(x)$ is the second order Legendre polynomial, $a$ and $b$ are parameters which define the deviation of the distribution function of the phonons moving in the given direction, from its equilibrium value. We note, that the parameters $a$ and $b$ are not independent. As equilibrium in the given direction is attained quickly, by fast three-phonon processes, there is a relation between the parameters $a$ and $b$ which can be written as follows (see, for example, Refs. 8, 10)

$$
b=-\frac{3}{2} a .
$$

Having integrated the left hand and right hand sides of Eq. (56) over all phonon energies, and taking (57) and (58) into account, we obtain the relaxation time which appears in the expression for the first viscosity,

$$
\tau^{-1}=-\frac{15}{2 \pi^{4}} \frac{c^{4}}{\left(k_{B} T\right)^{5}} \frac{\int I^{\prime}\left(p_{1}\right) p_{1}^{3} d p_{1}}{P_{2}\left(\cos \theta_{1}\right)},
$$

where, from (15)-(17), (54), (57), and (58), the collision integral can be written as 


$$
\begin{gathered}
I^{\prime}\left(p_{1}\right)=\frac{1}{2} \int d^{3} p_{2} d^{3} p_{3} d^{3} p_{4} W n_{1}^{(0)} n_{2}^{(0)}\left(1+n_{3}^{(0)}\right)\left(1+n_{4}^{(0)}\right) \times \\
\times\left\{\left(\varepsilon_{1}-\frac{3}{2} c p_{1}\right) P_{2}\left(\cos \theta_{1}\right)+\left(\varepsilon_{2}-\frac{3}{2} c p_{2}\right) P_{2}\left(\cos \theta_{2}\right)-\right. \\
\left.-\left(\varepsilon_{3}-\frac{3}{2} c p_{3}\right) P_{2}\left(\cos \theta_{3}\right)-\left(\varepsilon_{4}-\frac{3}{2} c p_{4}\right) P_{2}\left(\cos \theta_{4}\right)\right\} \times \\
\times \delta\left(\varepsilon_{\Sigma}\right) \delta\left(\mathbf{p}_{\Sigma}\right) .
\end{gathered}
$$

The probability density $W$, in Eq. (60), is defined by Eq. (4).

Equation (59) differs from that obtained in Ref. 12 for a nondecaying phonon spectrum, due to the terms in the curly brackets of the integrand in Eq. (60). They are the small terms containing $\psi\left(p_{i}\right)>0$, which occur in $\varepsilon_{i}$. As will be shown below, these terms determine the relaxation rate caused by phonons interacting at small angles (see result (75)). At small angles, the large terms in Eq. (60) are equal to zero and only the small terms containing $\psi(p)$ are left (see result (75)) .

In Eq. (59) for the relaxation time, which determines the coefficient of first viscosity, the integration can be made with the help of the $\delta$-functions, as in the third section. Further integration can only be precisely made numerically. The results of this integration are shown in Fig. 2 by curve 1 . However the contribution of large and small angles to Eq. (60) could be approximately calculated analytically; this will be done in the next section where we also discuss the results shown in Fig. 2.

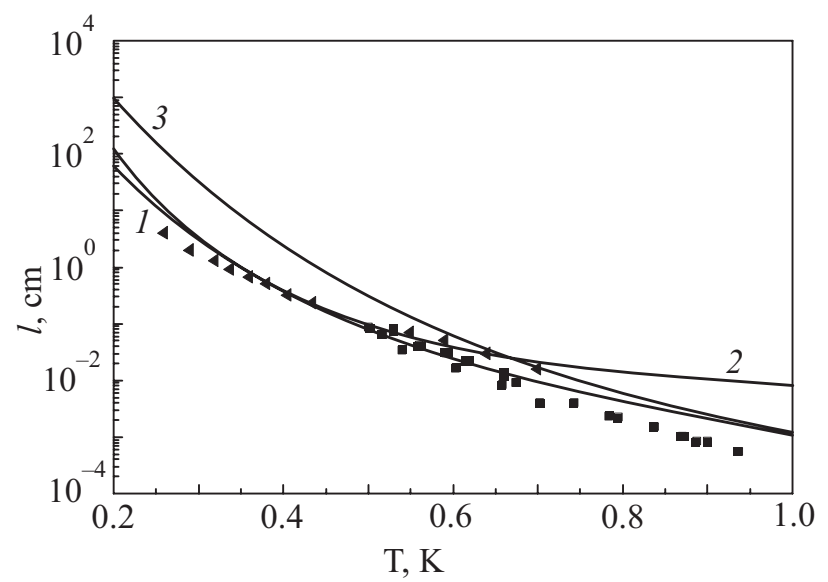

Fig. 2. The phonon mean free path $l=c \tau$ as a function of temperature $T$. Curve 1 is calculated from the relaxation time defined by Eq. (59); curve 2 represents Benin's result (see Ref. 7); curve 3 is a numerical calculation of the relaxation time from Eq. (59) without the contribution of small angles between $\mathbf{p}_{1}$ and $\mathbf{p}_{2}$ (i.e., the lower limit of integration over angles is equal to $30^{\circ}$ ); experimental points from Greywall [24] are marked by triangles, the squares show the experimental data from Zadorozhko et al. [25].

\section{Calculation of the contribution of small and large angle scattering to the relaxation time which deter- mines the coefficient of first viscosity}

To calculate the contribution of scattering at small angles between the interacting phonons in Eq. (60), as well as in the fourth section, we consider the contribution to Eq. (60) of the five main terms, which correspond to the five intermediate states I-V (see (6)).

We begin with the first intermediate state. The probability density of four-phonon process caused by the intermediate state I, at small angles between the momenta of the interacting phonons, is given by Eq. (36). After substituting Eq. (36) into Eq. (60) we obtain

$$
\begin{gathered}
I_{\mathrm{I}}^{\prime}\left(\mathbf{p}_{1}\right)=\frac{\hbar}{4} \frac{1}{(2 \pi \hbar)^{6}} \int d^{3} q d^{3} p_{2} d^{3} p_{3} d^{3} p_{4} \times \\
\times \frac{W\left(\mathbf{p}_{3}, \mathbf{p}_{4} \mid \mathbf{q}\right) W\left(\mathbf{q} \mid \mathbf{p}_{1}, \mathbf{p}_{2}\right)}{\Gamma_{d}(\mathbf{q})} \times\left\{\left(\varepsilon_{1}-\frac{3}{2} c p_{1}\right) P_{2}\left(\cos \theta_{1}\right)+\right. \\
+\left(\varepsilon_{2}-\frac{3}{2} c p_{2}\right) P_{2}\left(\cos \theta_{2}\right)-\left(\varepsilon_{3}-\frac{3}{2} c p_{3}\right) P_{2}\left(\cos \theta_{3}\right)- \\
\left.-\left(\varepsilon_{4}-\frac{3}{2} c p_{4}\right) P_{2}\left(\cos \theta_{4}\right)\right\} n_{1}^{(0)} n_{2}^{(0)}\left(1+n_{3}^{(0)}\right)\left(1+n_{4}^{(0)}\right) \times
\end{gathered}
$$$$
\times \delta\left(\mathbf{p}_{1}+\mathbf{p}_{2}-\mathbf{q}\right) \delta\left(\mathbf{p}_{3}+\mathbf{p}_{4}-\mathbf{q}\right) \delta\left(\varepsilon_{1}+\varepsilon_{2}-\varepsilon_{q}\right) \delta\left(\varepsilon_{3}+\varepsilon_{4}-\varepsilon_{q}\right) \text {. }
$$

The curly brackets in Eq. (61) can be rewritten in the form

$$
\left\{P_{2}\left(\cos \theta_{1}\right) A_{12 \mid q}^{1}+P_{2}\left(\cos \theta_{q}\right) A_{q \mid 34}^{q}\right\},
$$

where

$$
\begin{aligned}
A_{12 \mid q}^{1}= & \left(\varepsilon_{1}-\frac{3}{2} c p_{1}\right)+\left(\varepsilon_{2}-\frac{3}{2} c p_{2}\right) P_{2}\left(\cos \theta_{21}\right)- \\
& -\left(\varepsilon_{q}-\frac{3}{2} c q\right) P_{2}\left(\cos \theta_{q 1}\right), \\
A_{q \mid 34}^{q}= & \left(\varepsilon_{q}-\frac{3}{2} c q\right)-\left(\varepsilon_{3}-\frac{3}{2} c p_{3}\right) P_{2}\left(\cos \theta_{3 q}\right)- \\
& -\left(\varepsilon_{4}-\frac{3}{2} c p_{4}\right) P_{2}\left(\cos \theta_{4 q}\right),
\end{aligned}
$$

where $\theta_{i j}$ is the angle between phonons with momenta $\mathbf{p}_{i}$ and $\mathbf{p}_{j}$. The subscripts of $A$ indicates the corresponding process (for example, 12 $\mid q$ corresponds to $\mathbf{p}_{1}+\mathbf{p}_{2} \rightarrow \mathbf{q}$, the superscript shows the momentum direction to which all angles are referenced. We note, that for the $A$ quantities the following relations are valid

$$
A_{12 \mid q}^{1}=-A_{q \mid 12}^{1} ; \quad A_{q \mid 34}^{q}=-A_{34 \mid q}^{q} .
$$


When deriving Eq. (62) we used the result of the theorem about the addition of Legendre functions. This states that if two directions in space are given by their polar angles $\vartheta, \vartheta^{\prime}$ and their azimuth angles $\varphi, \varphi^{\prime}$ then the Legendre function of the $n$-th order, which depends on the cosine of the angle $\vartheta^{\prime \prime}$ between these directions, satisfies the following integral relation:

$$
\int_{0}^{2 \pi} P_{n}\left(\cos \vartheta^{\prime \prime}\right) d \varphi=2 \pi P_{n}(\cos \vartheta) P_{n}\left(\cos \vartheta^{\prime}\right) .
$$

Taking the above into account, the collision integral (61) can be written as

$$
\begin{gathered}
I_{\mathrm{I}}^{\prime}\left(\mathbf{p}_{1}\right)=\frac{\hbar}{4} \frac{1}{(2 \pi \hbar)^{6}} \int d^{3} q d^{3} p_{2} d^{3} p_{3} d^{3} p_{4} \times \\
\times \frac{W\left(\mathbf{p}_{3}, \mathbf{p}_{4} \mid \mathbf{q}\right) W\left(\mathbf{q} \mid \mathbf{p}_{1}, \mathbf{p}_{2}\right)}{\Gamma_{d}(\mathbf{q})} n_{1}^{(0)} n_{2}^{(0)}\left(1+n_{3}^{(0)}\right)\left(1+n_{4}^{(0)}\right) \times \\
\times\left\{P_{2}\left(\cos \theta_{1}\right) A_{12 \mid q}^{1}+P_{2}\left(\cos \theta_{q}\right) A_{q \mid 34}^{q}\right\} \times \\
\times \delta\left(\mathbf{p}_{1}+\mathbf{p}_{2}-\mathbf{q}\right) \delta\left(\mathbf{p}_{3}+\mathbf{p}_{4}-\mathbf{q}\right) \delta\left(\varepsilon_{1}+\varepsilon_{2}-\varepsilon_{q}\right) \delta\left(\varepsilon_{3}+\varepsilon_{4}-\varepsilon_{q}\right) .
\end{gathered}
$$

The integral from the second term in curly brackets is equal to zero because

$$
\int_{0}^{\pi} P_{2}\left(\cos \theta_{q}\right) \sin \theta_{q} d \theta_{q}=0 .
$$

Integration over $\mathbf{p}_{3}$ and $\mathbf{p}_{4}$ in Eq. (67) can be precisely made analytically. As a result, we have

$$
I_{\mathrm{I}}^{\prime}\left(\mathbf{p}_{1}\right)=-\frac{1}{2} \beta\left(p_{1}\right) P_{2}\left(\cos \theta_{1}\right),
$$

where

$$
\begin{aligned}
\beta\left(p_{1}\right)=\int & d^{3} q d^{3} p_{2} \frac{W\left(\mathbf{q} \mid \mathbf{p}_{1}, \mathbf{p}_{2}\right)}{(2 \pi \hbar)^{3}} n_{q}^{(0)}\left(1+n_{1}^{(0)}\right)\left(1+n_{2}^{(0)}\right) \times \\
& \times A_{q \mid 12}^{1} \delta\left(\mathbf{p}_{1}+\mathbf{p}_{2}-\mathbf{q}\right) \delta\left(\varepsilon_{1}+\varepsilon_{2}-\varepsilon_{q}\right) .
\end{aligned}
$$

Similarly for the remaining terms we have

$$
\begin{aligned}
& I_{\mathrm{II}}^{\prime}\left(\mathbf{p}_{1}\right)=I_{\mathrm{III}}^{\prime}\left(\mathbf{p}_{1}\right)=-\frac{1}{4} \xi\left(p_{1}\right) P_{2}\left(\cos \theta_{1}\right), \\
& I_{\mathrm{IV}}^{\prime}\left(\mathbf{p}_{1}\right)=I_{\mathrm{V}}^{\prime}\left(\mathbf{p}_{1}\right)=-\frac{1}{4} \beta\left(p_{1}\right) P_{2}\left(\cos \theta_{1}\right),
\end{aligned}
$$

where

$$
\begin{aligned}
\xi\left(p_{1}\right)=-\int & d^{3} q d^{3} p_{3} \frac{W\left(\mathbf{q}, \mathbf{p}_{3} \mid \mathbf{p}_{1}\right)}{(2 \pi \hbar)^{3}} n_{1}^{(0)}\left(1+n_{3}^{(0)}\right)\left(1+n_{q}^{(0)}\right) \times \\
& \times A_{1 \mid 3 q}^{1} \delta\left(\mathbf{p}_{1}-\mathbf{p}_{3}-\mathbf{q}\right) \delta\left(\varepsilon_{1}-\varepsilon_{3}-\varepsilon_{q}\right) .
\end{aligned}
$$

Substituting (69), (71)-(72) into (59), we obtain the relaxation time $\tau_{s m}$ due to the interaction of phonons at small angles

$$
\tau_{s m}^{-1}=\frac{15}{2 \pi^{4}} \frac{c^{4}}{\left(k_{B} T\right)^{5}} \int\left\{\frac{1}{2} \xi\left(p_{1}\right)+\beta\left(p_{1}\right)\right\} p_{1}^{3} d p_{1} .
$$

Integrating Eq. (74) (see Appendix B) we finally derive

$$
\begin{gathered}
\tau_{s m}^{-1}=\frac{45(u+1)^{2}}{2^{8} \pi^{5} \hbar^{4} \rho} \frac{c^{5}}{\left(k_{B} T\right)^{5}} \times \\
\times \int d p_{1} \int d p_{3} \frac{p_{1}^{2} p_{2}^{2} p_{3}^{2} \gamma\left(p_{1}, p_{3}\right)^{2} \eta\left(\gamma\left(p_{1}, p_{3}\right)\right)}{\sinh \frac{\varepsilon_{1}}{k_{B} T} \sinh \frac{\varepsilon_{2}}{k_{B} T} \sinh \frac{\varepsilon_{3}}{k_{B} T}}
\end{gathered}
$$

where

$$
\gamma\left(p_{1}, p_{3}\right)=p_{1} \psi\left(p_{1}\right)-p_{3} \psi\left(p_{3}\right)-\left(p_{1}-p_{3}\right) \psi\left(p_{1}-p_{3}\right)
$$

and $\eta(x)$ is the Heaviside function, which is equal to unity when $x \geq 0$ and to zero when $x<0$.

Equation (75) is identical with the result in Ref. 7 for the relaxation time of the second spherical harmonic, which defines the coefficient of the first viscosity. The time $\tau_{s m}$ from Eq. (75) is the same as $\tau_{\perp} / 6$ in Refs. 8, 9 . The presence of the numerical factor of $1 / 6$ is connected with the definition of the time of traverse relaxation which was given in Refs. 8, 9. However the coefficients of first viscosity are the same in our and their theories. Our relaxation time, given by Eq. (59) at small angles, automatically includes the process of transverse relaxation of Refs. 8 and 9.

At larger angles, the denominators of the matrix elements cease to be resonant. Then the matrix element actually ceases to depend on the exact form of the function $\psi(p)$. In this case, the integration of Eq. (60), with the approximate simplified matrix element, was made in Ref. 12 where the following relation for the $4 \mathrm{pp}$ rate was obtained

$$
\frac{1}{\tau_{4 p p}^{(L)}}=\frac{9 \cdot 13 !(u+1)^{4}}{2^{13}(2 \pi)^{7} \hbar^{7} \rho^{2} c^{10}}\left(k_{B} T\right)^{9} .
$$

Numerical calculations with the exact matrix element from Eq. (59), in the region of angles where $\theta_{12}>30^{\circ}$, gives practically the same numerical value for the relation time as that obtained from Eq. (77) when $T<0.7 \mathrm{~K}$. When $T>0.7 \mathrm{~K}$ the exact calculation of the relaxation time begins to deviate from the approximate relation for $\tau_{4 p p}^{(L)}$ (see (77)) to larger values of time, so that when $T=1 \mathrm{~K}$, the exact value of the relaxation time appears twice as large as the approximate time $\tau_{4 p p}^{(L)}$.

As a result, the rate $\tau^{-1}$ which defines the coefficient of the first viscosity is equal to the sum of the small-angle rate $\tau_{s m}^{-1}$ and of rate $\tau_{4 p p}^{-1}$ of the «exclusive» four-phonon 
processes which occur at larger angles between the scattering phonons

$$
\tau^{-1}=\tau_{s m}^{-1}+\tau_{4 p p}^{-1}
$$

Here $\tau_{s m}^{-1}$ is given by Eq. (75) and $\tau_{4 p p}^{-1}$ is obtained from Eq. (59) where we omit the integration on small angles and is rather well described by the analytical relation (77).

We see from Fig. 2, that at low temperatures $(T<0.5 \mathrm{~K})$, the main contribution to the viscosity is due to three-phonon processes. At higher temperatures $(T>0.9 \mathrm{~K})$, the situation changes and the contribution of the exclusive four-phonon processes predominates over the contribution of three-phonon processes. However in this temperature region, there is a considerable contribution to the viscosity coefficient from phonon-roton interactions and it is necessary to take these processes into account. The deviation of the experimental points $[24,25]$ from the theoretical curve at temperatures higher $0.7 \mathrm{~K}$ is due exactly to ignoring these phonon-roton interactions. In the intermediate temperature range (from 0.5 to $0.9 \mathrm{~K}$ ) the contributions of three-phonon and four-phonon relaxation have the same order of magnitude and both processes should be taken into account for calculating the first viscosity coefficient.

\section{The attenuation of a beam of h-phonons by ther- mal phonons}

In the experiment [13] a pulse of h-phonons was propagated through superfluid helium over different path lengths, to a detector. The amplitude of the pulse was measured as a function of the temperature $T$ of the liquid helium, in the range $0.07 \mathrm{~K} \leq T \leq 0.21 \mathrm{~K}$. The experimental data from Ref. 13 are shown in Fig. 3, the different sets of points are for different path lengths of the h-phonon signal.

The theory given above allows us to obtain an analytical description of the experiment [13]. As the phonons in the h-phonon pulse have energies close to $10 \mathrm{~K}$ (see, for example, Ref. 26), then our problem is reduced to finding the relaxation time of an h-phonon, with energy $10 \mathrm{~K}$, in an isotropic phonon system. The liquid He II at a given temperature $T$ is an isotropic phonon system described by the Bose distribution function for all momenta of phonons up to $p_{\max }$. The rate of relaxation $v_{h}$ is defined by Eq. (24) with $\chi=1$ and integration limits given by the relations

$$
\begin{gathered}
p_{3 \text { low }}=\frac{p_{1}+p_{2}}{2}, \quad p_{3 \text { up }}=\min \left(p_{1}+p_{2}, p_{\text {max }}\right), \\
p_{2 \text { low }}=0, \quad p_{\text {2up }}=p_{\text {max }},
\end{gathered}
$$

where $p_{\max }=20 \mathrm{~K}$ is the maximal momentum of phonons in liquid helium.

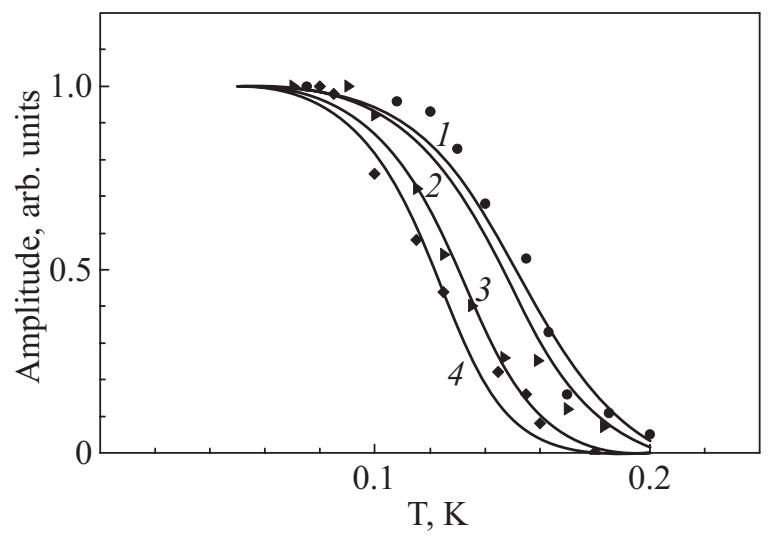

Fig. 3. The amplitude of the h-phonon signal as a function of temperature $T$. The solid curves show the results of the theoretical calculation: curve 1 corresponds to the propagation path length $l_{\mathrm{He}}=4 \mathrm{~mm}$, curve 2 to $5 \mathrm{~mm}$, curve 3 to $10.2 \mathrm{~mm}$ and curve 4 to $15.7 \mathrm{~mm}$. Experimental data from Ref. 13 at different values of the h-phonon path length in liquid helium are shown by the sets of points. Circles correspond to $l_{\mathrm{He}}=5 \mathrm{~mm}$, triangles to $10.2 \mathrm{~mm}$ and squares to the path length equal to $15.7 \mathrm{~mm}$.

If we know the rate $v_{h}$ for this process, and taking into account that h-phonons in a pulse have momentum close to $10 \mathrm{~K}$, we can calculate the attenuation coefficient $A$ of $\mathrm{h}$-phonons in an isotropic phonon system using the relation

$$
A=1-\exp \left(-\frac{l_{\mathrm{He}} v_{h}}{v_{c}}\right)
$$

Here $v_{h}$ is the relaxation rate (24) of phonons with energy of $10 \mathrm{~K}$, in an isotropic phonon system at temperature $T$ and $\chi=1, l_{\mathrm{He}}$ is the path length of the h-phonon in the liquid helium, and $v_{c}$ is the group velocity of the h-phonons.

The calculated attenuation coefficients for an h-phonon signal are shown in Fig. 3. The solid curves are for the different h-phonon path lengths, three of which were used in the experiment. We see from Fig. 3, that the calculated results from the analytic expressions (24) and (81) are in good agreement with the experimental data points of Ref. 13. In Ref. 13 the attenuation was calculated using a computer simulation with a simplified model matrix element. These calculations are not in quite such good agreement with the experimental data.

\section{Conclusion}

In this paper, we have investigated four-phonon scattering processes, in superfluid helium, when the phonon systems is isotropic. The matrix element (9) for four-phonon processes is derived for the whole range of momentum including the range where three-phonon processes are allowed. The problem is unusual in this range as the 
matrix element has resonances when the angle between the interacting phonons is small. We explain how to solve this problem.

The $4 p p$ scattering rate is found from the kinetic equation for phonons in liquid helium (24). The contribution of different angular groups of phonons to the $4 \mathrm{pp}$ rate is evaluated numerically (see Fig. 1). This analysis shows that the contribution of small angle scattering to this $4 \mathrm{pp}$ rate, is almost exactly the same as the $3 p p$ rate. Then it is shown analytically, that at small angles, the four-phonon process can be represented as two consecutive three-phonon processes, and moreover, that the rate of such processes is given by the rate of three-phonon processes. The contribution of larger angles to the scattering rate, using Eq. (24), gives the rate $v_{4 p p}$ of «exclusive» four-phonon processes. These processes exclude those $4 \mathrm{pp}$ scatterings that can be represented by $3 p p$ scattering.

From the matrix element expressed in Eq. (9), we derive the relaxation time (59) which appears in the expression for the coefficient of first viscosity of superfluid helium. The evaluation of the rate shows that is the same as the sum of the rate for traverse relaxation caused by three-phonon processes (75), and the rate for exclusive four-phonon processes (77) (see Fig. 2). It is shown that at low temperatures $(T<0.5 \mathrm{~K})$, the main contribution to the viscosity is given by three-phonon processes, and at higher temperature $(T>0.9 \mathrm{~K})$, the contribution of four-phonon processes is much larger than the contribution from three-phonon processes. In the intermediate range of temperatures $0.5 \mathrm{~K}<T<0.7 \mathrm{~K}$, both rates have the same order of magnitude and both processes give a significant contribution to the viscosity coefficient. We find generally very good agreement with the measured values of the viscosity in the temperature range where phonons are the dominant excitation, see Fig. 2. At higher temperatures, rotons begin to become increasingly important.

It is noted that the present analytical formulation automatically includes all the processes in transverse relaxation, which previously was treated as a separate mechanism in Refs. 8 and 9.

Finally we calculate the attenuation of an h-phonon pulse propagating through liquid helium at a finite temperature. The attenuation is due to the $\mathrm{h}$-phonons being scattered by the isotropic thermal phonons. The calculated the results are in good agreement with experimental data (see Fig. 3).

We conclude that the analysis in this paper gives a very good theoretical description of the dissipative relaxation of liquid helium which is only slightly perturbed from isotropic thermal equilibrium. We have shown that the theory gives a very good quantitative explanation of the measured values of viscosity and the attenuation of beams of high-energy phonons.
We are grateful to the EPSRC for supporting this work through the grant EP/F 019157/1.

\section{Appendix A: Calculation of the rate of four-phonon processes $v_{4 p p}$}

In the integrand of Eq. (23), without any loss of generality, we can choose the angle $\varphi_{1}$ as the computing origin of angles $\varphi_{i}$. In this case $\delta$-functions in Eq. (23) can be rewritten as

$$
\begin{aligned}
\delta\left(\mathbf{p}_{\Sigma}\right)=\delta\left(p_{1 \perp}\right. & \left.+p_{2 \perp} \cos \varphi_{2}-p_{3 \perp} \cos \varphi_{3}-p_{4 \perp} \cos \varphi_{4}\right) \times \\
\times \delta\left(p_{2 \perp}\right. & \left.\sin \varphi_{2}-p_{3 \perp} \sin \varphi_{3}-p_{4 \perp} \sin \varphi_{4}\right) \times \\
& \times \delta\left(p_{1 \|}+p_{2 \|}-p_{3 \|}-p_{4 \|}\right) \\
\delta\left(\varepsilon_{\Sigma}\right) & =\frac{1}{c} \delta\left(p_{1}+p_{2}-p_{3}-p_{4}-\phi\right) .
\end{aligned}
$$

Here $p_{i \perp}=p_{i} \sqrt{2 \zeta_{i}-\zeta_{i}^{2}}, p_{i \|}=p_{i}\left(1-\zeta_{i}\right)$ and $\phi$ is defined by Eq. (26).

In order to integrate Eq. (23) over $\varphi_{3}$ and $\varphi_{4}$ we introduce new variables

$$
\begin{aligned}
& X=p_{3 \perp} \cos \varphi_{3}+p_{4 \perp} \cos \varphi_{4}, \\
& Y=p_{3 \perp} \sin \varphi_{3}+p_{4 \perp} \sin \varphi_{4} .
\end{aligned}
$$

Taking Eqs. (84) and (85) into account, Eq. (23) can be rewritten as

$$
\begin{gathered}
v_{4 p p}=\frac{p_{1}}{2^{10} \pi^{5} \hbar^{7} \rho^{2}} \frac{1}{\left(1+n_{1}^{(0)}\right)} \times \\
\times \int \frac{d p_{2} d p_{3} d p_{4} d \zeta_{2} d \zeta_{3} d \zeta_{4} d \varphi_{2} d X d Y}{\sqrt{4 p_{3 \perp}^{2} p_{4 \perp}^{2}-\left(X^{2}+Y^{2}-p_{3 \perp}^{2}-p_{4 \perp}^{2}\right)^{2}}} \times \\
\times M_{\Sigma}^{2} \delta\left(\mathbf{p}_{\Sigma}\right) \delta\left(\varepsilon_{\Sigma}\right) n_{2}^{(0)}\left(1+n_{3}^{(0)}\right)\left(1+n_{4}^{(0)}\right) p_{2}^{3} p_{3}^{3} p_{4}^{3} .
\end{gathered}
$$

Here and below the integration is made so that radicands are nonnegative.

As $M_{\Sigma}$ depends on $\cos \varphi_{3}$ and $\cos \varphi_{4}$, then to make the integration over $\varphi_{3}$ and $\varphi_{4}$, it is necessary to solve the set of equations

$$
\left\{\begin{array}{l}
p_{1 \perp}+p_{2 \perp} \cos \varphi_{2}-p_{3 \perp} \cos \varphi_{3}-p_{4 \perp} \cos \varphi_{4}=0 \\
p_{2 \perp} \sin \varphi_{2}-p_{3 \perp} \sin \varphi_{3}-p_{4 \perp} \sin \varphi_{4}=0
\end{array}\right.
$$

with regard to $\cos \varphi_{3}$ and $\cos \varphi_{4}$.

The set of Eqs. (87) has two solutions which can be written as

$$
\begin{gathered}
\cos \varphi_{3}^{( \pm)}= \\
=\frac{\left(p_{1 \perp}+p_{2 \perp} \cos \varphi_{2}\right)\left(A+p_{3 \perp}^{2}-p_{4 \perp}^{2}\right) \pm p_{2 \perp} \sin \varphi_{2} \sqrt{R}}{2 A p_{3 \perp}}
\end{gathered}
$$




$$
\begin{gathered}
\cos \varphi_{4}^{( \pm)}= \\
=\frac{\left(p_{1 \perp}+p_{2 \perp} \cos \varphi_{2}\right)\left(A-p_{3 \perp}^{2}+p_{4 \perp}^{2}\right) \mp p_{2 \perp} \sin \varphi_{2} \sqrt{R}}{2 A p_{4 \perp}} .
\end{gathered}
$$

Here

$$
\begin{gathered}
R=4 p_{3 \perp}^{2} p_{4 \perp}^{2}-\left(A-p_{3 \perp}^{2}-p_{4 \perp}^{2}\right)^{2}, \\
A=p_{1 \perp}^{2}+p_{2 \perp}^{2}+2 p_{1 \perp} p_{2 \perp} \cos \varphi_{2} .
\end{gathered}
$$

Making the integration in (86) with a help $\delta$-functions (82), (83) over $\varphi_{3}, \varphi_{4}$ and $p_{4}, \zeta_{4}$ we obtain Eq. (24).

\section{Appendix B: Derivation of $\tau_{s m}^{-1}$}

For the further integration of Eq. (74) it is convenient to symmetrize Eq. (70) for $\beta\left(p_{1}\right)$ :

$$
\beta\left(p_{1}\right)=\frac{\beta\left(p_{1}\right)+\widetilde{\beta}\left(p_{1}\right)}{2} .
$$

Here $\widetilde{\beta}\left(p_{1}\right)$ is derived from $\beta\left(p_{1}\right)$ by interchanging $\mathbf{q} \leftrightarrow \mathbf{p}_{2}$ in the integrand of Eq. (70). Nevertheless $\widetilde{\beta}\left(p_{1}\right)$ is of course equal to $\beta\left(p_{1}\right)$.

As the integrand of Eq. (74) does not depend on $\varphi_{1}$ and $\theta_{1}$, we can rewrite Eq. (74) taking (91) into account as

$$
\tau_{s m}^{-1}=\frac{15}{16 \pi^{5}} \frac{c^{4}}{\left(k_{B} T\right)^{5}} \int\left\{\xi\left(p_{1}\right)+\beta\left(p_{1}\right)+\widetilde{\beta}\left(p_{1}\right)\right\} p_{1} d^{3} p_{1} .
$$

The integral in Eq. (92) can be represented as a sum of three integrals

$$
I_{\tau}=\int \xi\left(p_{1}\right) p_{1} d^{3} p_{1}+\int \beta\left(p_{1}\right) p_{1} d^{3} p_{1}+\int \widetilde{\beta}\left(p_{1}\right) p_{1} d^{3} p_{1} .
$$

We replace the variables $\xi\left(p_{1}\right), \beta\left(p_{1}\right), \widetilde{\beta}\left(p_{1}\right)$ by their expressions. After that we replace $\mathbf{q}$ with $\mathbf{p}_{2}$ in the first integral, in the second and third integrals we replace $\mathbf{q}$ with $\mathbf{p}_{3}$. As a result, the integration in all three integrals will be over variables $\mathbf{p}_{1}, \mathbf{p}_{2}$ and $\mathbf{p}_{3}$. Further, in the second integral we rename $\mathbf{p}_{1}$ and $\mathbf{p}_{3}$ and in the third we rename $\mathbf{p}_{1}$ and $\mathbf{p}_{2}$. Thus in the second integral all angles will be counted off $\mathbf{p}_{3}$ and in the third counted off $\mathbf{p}_{2}$. As a result of all the above mentioned transformations, we obtain

$$
\begin{gathered}
I_{\tau}=\int \frac{W\left(\mathbf{p}_{1} \mid \mathbf{p}_{2}, \mathbf{p}_{3}\right)}{(2 \pi \hbar)^{3}} \times \\
\times \frac{\delta\left(\mathbf{p}_{1}-\mathbf{p}_{2}-\mathbf{p}_{3}\right) \delta\left(\varepsilon_{1}-\varepsilon_{2}-\varepsilon_{3}\right)}{8 \sinh \frac{\varepsilon_{1}}{k_{B} T} \sinh \frac{\varepsilon_{2}}{k_{B} T} \sinh \frac{\varepsilon_{3}}{k_{B} T} \Delta d^{3} p_{3} d^{3} p_{2} d^{3} p_{1},}
\end{gathered}
$$

$$
\begin{gathered}
\Delta=p_{1}\left\{-\left(\varepsilon_{1}-\frac{3}{2} c p_{1}\right)+\left(\varepsilon_{2}-\frac{3}{2} c p_{2}\right) P_{2}\left(\cos \theta_{12}\right)+\right. \\
\left.+\left(\varepsilon_{3}-\frac{3}{2} c p_{3}\right) P_{2}\left(\cos \theta_{13}\right)\right\}+p_{3}\left\{\left(\varepsilon_{1}-\frac{3}{2} c p_{1}\right) P_{2}\left(\cos \theta_{13}\right)-\right. \\
\left.-\left(\varepsilon_{3}-\frac{3}{2} c p_{3}\right)-\left(\varepsilon_{2}-\frac{3}{2} c p_{2}\right) P_{2}\left(\cos \theta_{23}\right)\right\}+p_{2}\left\{\left(\varepsilon_{1}-\frac{3}{2} c p_{1}\right) \times\right. \\
\left.\times P_{2}\left(\cos \theta_{12}\right)-\left(\varepsilon_{2}-\frac{3}{2} c p_{2}\right)-\left(\varepsilon_{3}-\frac{3}{2} c p_{3}\right) P_{2}\left(\cos \theta_{23}\right)\right\} .
\end{gathered}
$$

We make the integration in Eq. (94) on $\mathbf{p}_{2}$ and $\zeta_{3}$ with a help of $\delta$-functions. As the integrand does not depend on angles, then the integration over the remaining angles is trivial. Finally for the rate $\tau_{s m}^{-1}$ we obtain Eq. (75).

1. A.F.G. Wyatt, N.A. Lockerbie, and R.A. Sherlock, J. Phys. Condens. Matter 1, 3507 (1989).

2. I.N. Adamenko, K.E. Nemchenko, A.V. Zhukov, M.A.H. Tucker, and A.F.G. Wyatt, Phys. Rev. Lett. 82, 1482 (1999).

3. A.F.G. Wyatt, M.A.H. Tucker, I.N. Adamenko, K.E. Nemchenko, and A.V. Zhukov, Phys. Rev. B62, 9402 (2000).

4. I.N. Adamenko, Yu.A. Kitsenko, K.E. Nemchenko, V.A. Slipko, and A.F.G. Wyatt, Fiz. Nizk. Temp. 31, 607 (2005) [Low Temp. Phys. 31, 459 (2005)].

5. I.N. Adamenko, Yu.A. Kitsenko, K.E. Nemchenko, V.A. Slipko, and A.F.G. Wyatt, Phys. Rev. B68, 134505 (2006).

6. H.J. Maris, Phys. Rev. A8, 1980 (1973).

7. D. Benin, Phys. Rev. B11, 145 (1975).

8. V.L. Gurevich and B.D. Laikhtman, JETP 69, 1230 (1975).

9. Yu.A. Kosevich, Fiz. Nizk. Temp. 9, 479 (1983) [Low Temp. Phys. 9, 242 (1983)].

10. Yu.A. Matveev and I.M. Khalatnikov, JETP Lett. 22, 581 (1975).

11. L.D. Landau and I.M. Khalatnikov, JETP 19, 637 (1949).

12. L.D. Landau and I.M. Khalatnikov, JETP 19, 709 (1949).

13. M.A.H. Tucker and A.F.G. Wyatt, J. Phys.: Condens. Matter 4, 7745 (1992).

14. R. Vovk, C.D.H. Williams, and A.F.G. Wyatt, Phys. Rev. Lett. 91, 235302 (2003).

15. D.H.S. Smith, R. Vovk, C.D.H. Williams, and A.F.G. Wyatt, Phys. Rev. B72, 054506 (2005).

16. D.H.S. Smith, C.D.H. Williams, and A.F.G. Wyatt, New J. Phys. 9, 52 (2007).

17. I.M. Khalatnikov, An Introduction to the Theory of Superfluidity, Addison-Wesley, Redwood City, CA (1989).

18. I.N. Adamenko, K.E. Nemchenko, and A.F.G. Wyatt, J. Low Temp. Phys. 125, 1 (2001).

19. V.M. Apalkov and M.E. Portnoi, Phys. Rev. B65, 125310 (2002).

20. V.M. Apalkov and M.E. Portnoi, Phys. Rev. B66, 121303 (R) (2002).

21. L.D. Landau and E.M. Lifshitz, Quantum Mechanics: Non-Relativistic Theory, Volume 3, Butterworth-Heinemann (1981). 
22. I.N. Adamenko, Yu.A. Kitsenko, K.E. Nemchenko, V.A. Slipko, and A.F.G. Wyatt, Fiz. Nizk. Temp. 33, 523 (2007) [Low Temp. Phys. 33, 387 (2007)].

23. I.N. Adamenko, Yu.A. Kitsenko, K.E. Nemchenko, V.A. Slipko, and A.F.G. Wyatt, Phys. Rev. B72, 054507 (2005).

24. D.S. Greywall, Phys. Rev. B23, 2152 (1981).
25. A.A. Zadorozhko, G.A. Sheshin, E.Ya. Rudavskii, V.K. Chagovets, and Yu.A. Kitsenko, Fiz. Nizk. Temp. 35, 134 (2009).

26. I.N. Adamenko, K.E. Nemchenko, V.A. Slipko, and A.F.G. Wyatt, Phys. Rev. B69, 144525 (2004). 